\title{
Epidemiological and clinical characteristics of 40 cases of COVID-19 outside Hubei Province, China
}

\author{
Ting $\mathrm{GAO}^{1}$, Yangling $\mathrm{XU}^{2}$, Xiaopeng $\mathrm{HE}^{1 *}\left(\mathrm{D}\right.$, Xiaofeng $\mathrm{XU}^{1}$, Lizhen $\mathrm{WANG}^{2}$, Yindi JIANG ${ }^{3}$, Changgang $\mathrm{WU}^{2}$, \\ Wenming ZHANG ${ }^{2}$
}

\begin{abstract}
To explore characteristics of patients with pneumonia infected by 2019 Novel Coronavirus (COVID-19) in 2019 outside Hubei Province, China. 40 patients with pneumonia infected by COVID-19 which were confirmed by COVID-19 nucleic acid test were included. Procalcitonin (PCT), serum amyloid A (SAA), C-reactive protein (CRP) and computed tomography (CT) manifestations were analyzed. $40 \%$ of patients had clear contact history with Wuhan or other areas of Hubei Province. $60 \%$ of patients were clustered diseases and $40 \%$ were imported cases. $75 \%$ of patients had initial fever, $7.5 \%$ had cough, $5 \%$ had sore throat at first. $45 \%$ had decreased lymphocyte count, $72.5 \%$ and 55\% patients had increased levels of SAA and CRP. $72.5 \%$ of the patients showed multiple ground glass lesions in one or two lungs on chest CT. $90 \%$ of the patients with pneumonia are of the common type, and $\alpha$-interferon atomization inhalation combined with Lopinavir/Ritonavir tablets were given to patients during treatment. $62.5 \%$ of the patients were treated with antibiotics, and $15 \%$ with hormone. All patients improved after treatment, and 14 patients were cured and discharged. Family cluster infection and asymptomatic infection may be the main way of spreading of COVID-19 pneumonia outside Hubei Province in China.
\end{abstract}

Keywords: 2019 Novel Coronavirus Pneumonia; Epidemiology; Clinical characteristics; Outside Hubei Province.

Pratical Application: Epidemiological and clinical characteristics of 40 cases of COVID-19 outside Hubei Province.

\section{Introduction}

Coronaviruses can cause multiple system infections in various animals and mainly respiratory tract infections in humans, such as severe acute respiratory syndrome (SARS) and Middle East respiratory syndrome (MERS). Most patients have mild symptoms and good prognosis. In December 2019, a group of new cases of pneumonia with unknown cause appeared in Wuhan City, Hubei Province, China. On January 3, 2020, a new coronavirus was found in the macrogene sequencing of bronchoalveolar lavage fluid in an infected patient, and it was subsequently named COVID-19 by WHO. At first, it was thought that COVID-19 might be related to zoonosis and environmental exposure in South China seafood market in Wuhan (Zhu et al., 2020). Human-to-human transmission is one of the main reasons for the rapid spread of the pneumonia infected with COVID-19 (Li et al., 2020; Huang et al., 2020). So far, a few patients with $2019 \mathrm{nCoV}$ have developed severe pneumonia, pulmonary oedema, ARDS, or multiple organ failure and have died. At present, the disease has spread to the whole China and many countries in the world (Lu et al., 2020a; Bogoch et al., 2020). As of 17:51, February 16, 2020, the Chinese government has notified a total of 68,592 patients confirmed pneumonia with COVID-19 and 1,666 dead patients. The reports of this disease are mainly concentrated in Hubei Province, especially in Wuhan City (Chen et al., 2020b), but there are few reports on the epidemiology and clinical characteristics of the cases outside Hubei Province. Therefore, the purpose of this study was to investigate the epidemiology and clinical manifestations of 40 patients diagnosed with COVID-19 from January 21, 2020 to February 16, 2020 in Xianyang Central Hospital of Shaanxi Province and Liaocheng infectious disease hospital of Shandong Province.

\section{Materials and methods}

\subsection{Subjects}

This study was approved by the ethics committee of Xianyang Central Hospital and Liaocheng infectious disease hospital (batch number: 20200205, 2020003). Written informed consent was obtained from patients involved before enrolment when data were collected retrospectively. A total of 40 patients diagnosed with pneumonia infected by COVID-19 from January 21, 2020 to February 16, 2020 in Xianyang Central Hospital and Liaocheng infectious disease hospital were enrolled in this retrospective, multicenter study. Among them, 17 patients were from Xianyang Central Hospital and 23 were from Liaocheng infectious disease hospital. Inclusion criteria: (1) suspected cases of covid-19 pneumonia; (2) sputum, throat swab, lower respiratory tract secretion and other samples were detected to be covid-19 nucleic acid positive by RT-PCR. The epidemiological 
data, demographics, signs and symptoms on admission were collected. All patients underwent CT.

\subsection{Diagnostic criteria}

A novel coronavirus pneumonia diagnosis and treatment plan developed by the general office of National Health Committee of China (the fifth revised version) was used in this study (Novel coronavirus pneumonia diagnosis and treatment plan (revised version fifth), 2020). Diagnostic criteria: (1) epidemiological history: within 14 days before the onset of the disease, there was travel history or residential history of the case in Wuhan and surrounding areas or local communities. Within 14 days before the onset of the disease, patients with fever or respiratory symptoms from Wuhan and surrounding areas or local communities were contacted. Within 14 days before the onset of the disease, there was a history of contact with people infected with new coronavirus. A novel coronavirus infection is a positive test of pathogenic nucleic acid. There is aggregation. (2) Clinical manifestations: fever or / and respiratory symptoms; with the imaging characteristics of pneumonia; found early normal or reduced white blood cells, or lymphocyte count. If patients had any one of the epidemiological history and any two of the clinical manifestations, it can be diagnosed as a suspected case.

\subsection{Clinical classification}

(1) Mild type. The clinical symptoms were mild, and there was no sign of pneumonia on imaging. (2) Ordinary type. There were fever, respiratory tract and other symptoms, and pneumonia was found by imaging. (3) Heavy type. Those who meet one of the following conditions: (a) Respiratory distress with $\mathrm{RR} \geq 30$ times/min; (b) In resting state, oxygen saturation $\leq 93 \%$; (c) Arterial partial pressure of oxygen $(\mathrm{PaO} 2) /$ concentration of oxygen $(\mathrm{FiO} 2) \leq 300 \mathrm{MMHG}(1 \mathrm{mmhg}=0.133 \mathrm{kpa})$. (d) Those who meet one of the following conditions: (4) Severe type. Those who meet one of the following conditions: (a) Respiratory failure and need mechanical ventilation; (b) Shock; (c) Other organ failure need ICU monitoring and treatment.

\subsection{Nucleic acid detection}

The nucleic acid of COVID-19 was detected by RT-PCR with nucleic acid test kit (Shanghai Jienuo biological Co., Ltd., Shanghai, China) in accordance with the manufacture's protocol. Results interpretation: the result was positive when cut off value was 40 and CT value was less than 37; the result was when CT value was more than 40; if CT value was between 37 and 40, it was gray area, and patients needed to be retested.

\subsection{Blood biochemical indexes}

Peripheral blood of patients was collected at admission. The levels of leukocyte, procalcitonin (PCT), serum amyloid A (SAA), C-reactive protein (CRP), and lymphocyte count were detected. Normal range of leukocyte: $(3.5-9.5) \times 10^{9} / \mathrm{L}$; normal range of lymphocyte count: $(1.0-3.0) \times 10^{\%} / \mathrm{L}$; normal range of PCT: $0-0.5 \mathrm{ng} / \mathrm{ml}$; normal range of SAA: $0-10 \mathrm{mg} / \mathrm{L}$; normal range of CRP: $0-10 \mathrm{mg} / \mathrm{L}$.

\subsection{Discharge standard}

At the same time, the following three conditions were met: (1) the temperature returned to normal for more than three days, and the respiratory symptoms improved significantly; (2) the pulmonary imaging showed that the inflammation was obviously absorbed; (3) Negative nucleic acid detection of respiratory pathogens for 2 consecutive times (sampling interval of at least $1 \mathrm{~d}$ ).

\subsection{Statistical analysis}

Statistical analysis was made by software SPSS17.0 (International Business Machines, corp., Armonk, NY, USA). The counting data were expressed as number (percentage), and the measurement data were expressed as means \pm standard deviation (SD). Significant differences between groups were assessed by independent sample t test. Differences were considered statistically significant when $P<0.05$.

\section{Results}

\subsection{Epidemiology}

Among 40 patients, 16 (40\%) had a clear contact history with people in Wuhan or other areas in Hubei Province (14 in Xianyang Central Hospital and 2 in Liaocheng infectious disease hospital), and the other 24 (60\%) had no clear exposure history. In addition, 16 cases (40\%) were imported and 24 cases $(60 \%)$ were clustered.

There were mainly imported patients in Liaocheng infectious disease hospital. Among the 17 diagnosed patients of COVID-19, 14 had a clear contact history with people in Wuhan or other areas in Hubei Province. Among them, 8 returned to work in Wuhan, 4 were from Wuhan to visit relatives with their relatives, 1 returned from school in Wuhan, and 1 attended a short-term meeting in Wuhan. In addition, one patient went to Wuhan to attend the meeting before the onset of the disease. After returning, a nucleic acid test was performed on him and he was diagnosed as positive of COVID-19, but no disease was found in the other participants within 14 days after isolation. 3 patients $(17.6 \%)$ had no obvious contact history of Hubei nationality (1 patient returned home from Nanjing without history of living in other places, and fever occurred on the 5th day after returning; 1 patient was a salesman in the local market, and there was no confirmed case after 14 days of medical isolation among the staff in the market; 1 patient had a history of gathering activities before the onset of the disease, and there was no Wuhan or Hubei nationality in the gathering staff). Among the 17 patients, there was one family clustering disease. The infected person was the grandson of Wuhan native who went home with his parents to visit his relatives. The infected person was his grandfather and grandmother. His parents and younger sister had fever and pneumonia. They had three times of COVID-19 nucleic acid tests and the results were negative. They were treated in the isolation area and the condition was stable.

In Liaocheng infectious disease hospital., 21 of the 23 cases diagnosed as COVID-19 were clustered diseases. Nine patients 
were colleagues and they came from the same supermarket, and 12 patients came from five families. The other 2 cases were imported cases. One case had a business trip in Wuhan before the onset of the disease. The other had lived in Jingzhou City, Hubei Province for a long time and returned to Liaocheng for family visit.

\subsection{Clinical characteristics}

Among the 40 patients, the longest time from onset to seek medical advice was 13 days, and the shortest was 1 day. The average consultation time was $(4.88 \pm 3.52)$ d. $16(40 \%)$ patients had underlying disease, including 4 with type 2 diabetes, 2 with hypertension, and 2 with a history of malignant tumors (1 with primary infection and 1 with non-Hodgkin's lymphoma). Two patients had a history of chronic hepatitis B, and two patients had a history of surgery (both were greater than 2 years). The average temperature of 40 patients was ( $37.6 \pm 0.7)$ ${ }^{\circ} \mathrm{C}$. Among them, 30 patients (75\%) first diagnosed as fever (21 patients with body temperature between $37.3^{\circ} \mathrm{C}$ and $38.2^{\circ} \mathrm{C}$, 9 patients with body temperature $\left.\geq 38.3^{\circ} \mathrm{C}\right) ; 3$ patients $(7.5 \%)$ first diagnosed as cough, all of which were dry cough; 2 patients (5\%) first diagnosed as pharyngalgia, and 1 patient (2.5\%) later developed fever; 1 patient (2.5\%) first diagnosed as headache, and later detected as normal body temperature; 4 patients (1\%) did not have obvious symptoms at the first diagnosis with exposure history, and a patient later fever.

\subsection{The levels of blood biochemical indexes}

Among 40 patients, 9 patients (22.5\%) had decreased levels of peripheral blood leukocyte and 31 patients (77.5\%) had normal leucocyte count. Peripheral blood lymphocyte count was decreased in 18 patients (45\%), which was normal in 22 patients (55\%). The levels of SAA were elevated in $29(72.5 \%)$ patients, and the levels of SAA were normal in 11 (27.5\%) patients. 22 (55\%) patients had elevated CRP levels, and 18 (45\%) patients had normal CRP levels. There were 3 (7.5\%) patients with elevated PCT levels and 37 (92.5\%) patients with normal PCT levels. The results were shown in Table 1.

\subsection{Nucleic acid test results}

Respiratory tract samples were collected from 40 patients with pharyngeal swabs, and all patients were tested for pathogenic nucleic acid at least twice. Sampling time must be at least 1 day apart. Among them, 37 (92.5\%) patients were positive at the first test; 2 (5\%) patients were positive at the second test; 1 (2.5\%) patient was weak positive at the first test and positive at the second test. Among 40 patients, 37 had pneumonia on chest $\mathrm{CT}$ at the time of diagnosis.

\subsection{Chest CT results}

Chest CT was performed on all 40 patients at admission. Three patients (7.5\%) showed no abnormalities in chest imaging at the beginning of the onset, and continued to have no abnormalities in the imaging examination at the later stage. Three patients (7.5\%) showed chordal shadow and ground glass change under pleura; five patients (12.5\%) showed grid like
Table 1. blood biochemical indexes in patients with COVID-19.

\begin{tabular}{lccc}
\hline \multicolumn{1}{c}{ Indexes } & Cases & Values & $P$ values \\
\hline PCT $(\mathrm{ng} / \mathrm{mL})$ & & & \\
$\geq 0.5$ & 3 & $1.37 \pm 0.81$ & 0.000 \\
$<0.5$ & 37 & $0.19 \pm 0.10$ & \\
SAA $(\mathrm{mg} / \mathrm{L})$ & & & \\
$\geq 10$ & 29 & $147.38 \pm 135.47$ & 0.000 \\
$<10$ & 11 & $6.02 \pm 1.85$ & \\
CRP $(\mathrm{mg} / \mathrm{L})$ & & & \\
$\geq 10$ & 22 & $42.78 \pm 61.59$ & 0.019 \\
$<10$ & 18 & $2.40 \pm 1.72$ & \\
Leucocyte count $(109 / \mathrm{L})$ & & & \\
$\geq 4$ & 31 & $6.05 \pm 1.79$ & 0.030 \\
$<4$ & 9 & $2.93 \pm 0.43$ & \\
Lymphocyte count $(109 / \mathrm{L})$ & & & \\
$\geq 1$ & 22 & $1.69 \pm 0.44$ & 0.018 \\
$<1$ & 18 & $0.63 \pm 0.22$ & \\
\hline
\end{tabular}

change and ground glass shadow of double lung; 12 patients (30\%) showed unilateral pneumonia or localized ground glass nodular shadow; 17 patients (42.5\%) showed multiple ground glass like change of double lung. The results were shown in Figure 1. The coincidence rate of chest CT was $92.5 \%$ with nucleic acid test.

\subsection{Clinical treatment and prognosis}

There were 3 mild type patients (7.5\%, 1 in Xianyang City, 2 in Liaocheng City), 36 ordinary type patients (90\%, 16 in Xianyang City, 1 in Liaocheng City), and 1 severe type patient (2.5\%, Liaocheng City). All patients were given IFN - $\alpha$ aerosol inhalation (5 million $\mathrm{U}$ or equivalent dose per time for adults, $2 \mathrm{ml}$ of sterile water were used for injection, twice a day) combined with Lopinavir/Ritonavir $(200 \mathrm{mg} / 50 \mathrm{mg}, 2$ pills each time, twice a day). 11 patients (27.5\%, 10 in Xianyang City, 1 in Liaocheng City) were given abidol tablets ( $0.2 \mathrm{~g}, 3$ times/day). 8 patients (20\%, Xianyang City) were given oseltamivir capsules (75mg, 2 times/day). 16 patients (40\%, 6 in Xianyang City and 10 in Liaocheng City) received ribavirin injection, an intravenous antiviral drug ( $0.5 \mathrm{~g}, 1$ time/d). 25 patients $(62.5 \%$, 4 in Xianyang City, 21 in Liaocheng City) were treated with antibiotics, mainly including fluoroquinolones and macrolides. Six patients (15\%, 3 in Xianyang city and 3 in Liaocheng City) were given intravenous hormone therapy (methylprednisolone $40-80 \mathrm{mg} /$ time, twice/d). One patient $(2.5 \%, 1$ in Xianyang City) was given high flow oxygen through nose to stabilize decreased blood oxygen saturation due to chest tightness and aggravation of shortness of breath. One patient $(2.5 \%, 1$ in Liaocheng City) have rapidly developed condition due to lung disease, and he was considered to have "inflammatory storm" reaction in consultation. After hemofiltration treatment, the patient's condition was further aggravated and transferred to the superior hospital for treatment. After treatment, 26 patients improved (65\%, 11 in Xianyang City, 15 in Liaocheng City), 14 patients were discharged (35\%, 6 in Xianyang City, 8 in Liaocheng City), and no one died. 


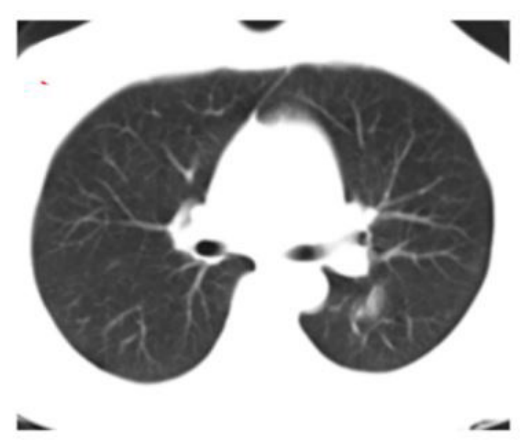

A

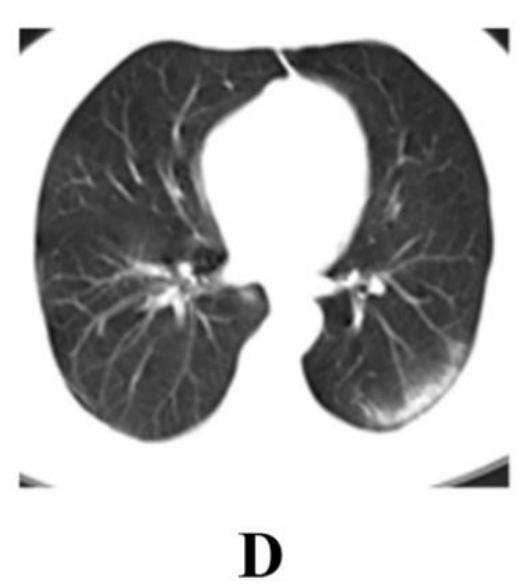

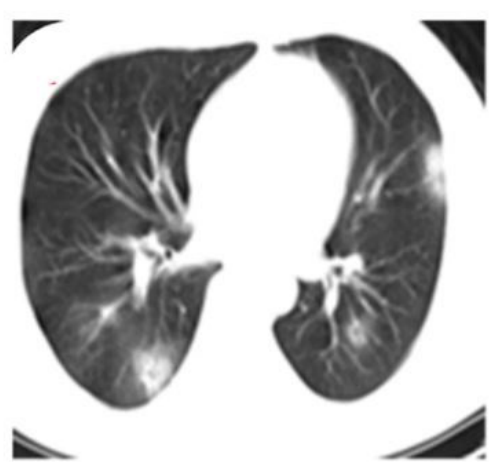

B

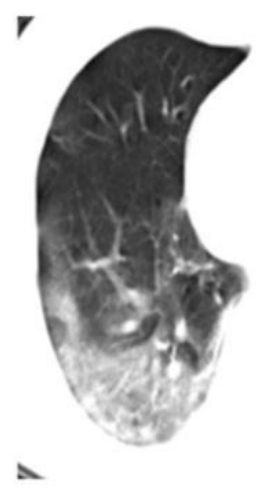

$\mathbf{E}$
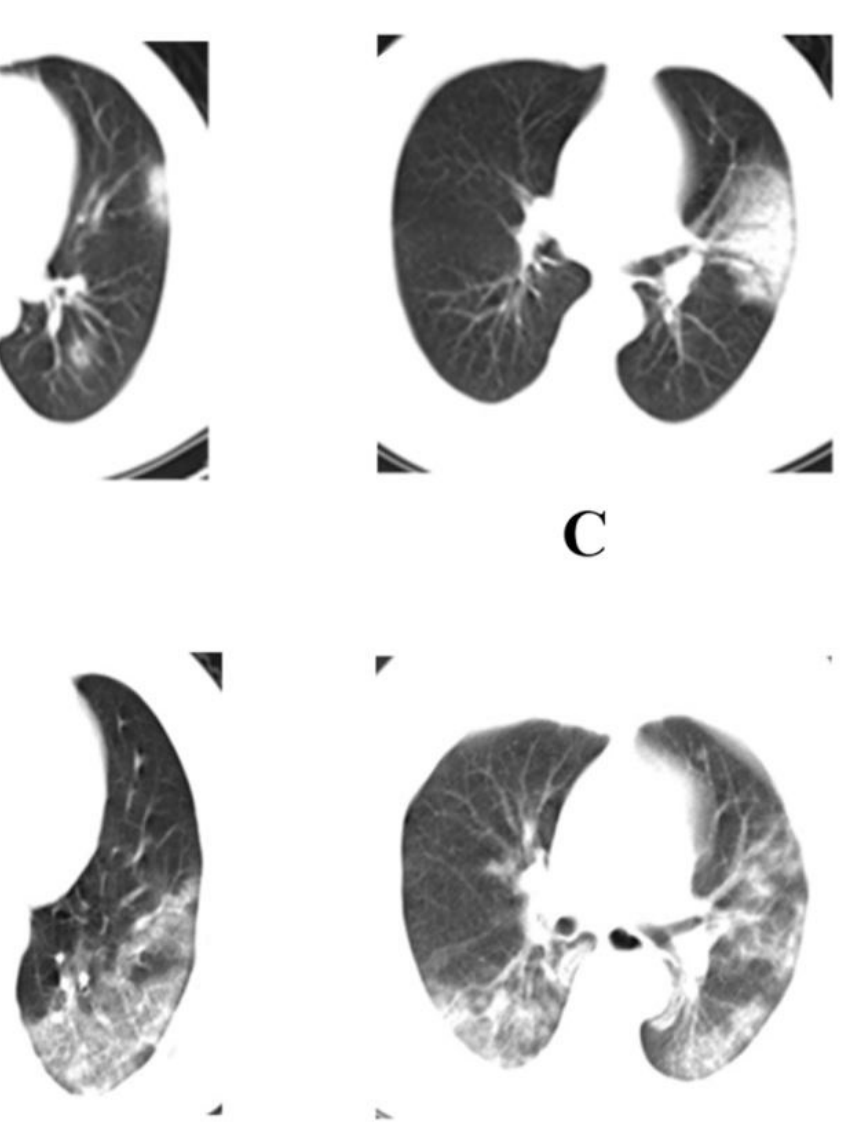

F

Figure 1. Typical imaging manifestations of patients with COVID-19. (A) Single shot ground glass shadow (GGO). (B) Multiple ground-glass opacity. (C) Single lung lamellar exudation shadow and bronchiectasis. (D) Single subpleural strip shadow and exudation shadow. (E) No double lung interstitial exudation shadow. (F) Diffuse ground glass shadow of both lungs.

\section{Discussion}

Coronavirus is a non-fragment positive RNA virus, which is widely distributed in human and other mammals. At present, there are six kinds of coronaviruses known to infect human beings, most of which cause mild symptoms of infection. However, severe acute respiratory syndrome (SARS)-COV and Middle East respiratory syndrome (MERS)-COV in $\beta$-coronavirus can cause acute respiratory syndrome and even death, and about 10000 people have died in the past (Kuiken et al., 2003; Drosten et al., 2003). The whole genome sequencing showed that the homology of SARS-COV-2 and SARS-COV was $79.5 \%$, and that of bat coronavirus was $96 \%$. The initial infected patients are mainly closely related to the South China seafood market in Wuhan. However, with the increase of the number of cases outside Wuhan, and most of them are imported cases, the phenomenon of human-to-Human transmission has become very clear (Li et al., 2020).

In a large sample study of patients with COVID-19, 71.8\% had a clear contact history with people in Wuhan or other areas of Hubei Province. In this study, only 40\% with COVID-19 had a clear contact history with people in Wuhan and other areas in Hubei Province, $82.3 \%$ of which are imported cases in Xianyang City, while only $8.7 \%$ in Liaocheng City. It may be related to the geographical location, transportation and personnel flow of the two cities. However, $91.3 \%$ of the patients with COVID-19 in Liaocheng City and 17.6\% in Xianyang City showed that COVID-19 was generally susceptible, suggesting that COVID-19 could be actively transmitted outside Hubei Province. Those results were similar with a previous study. In addition, there was $1 \%$ of the asymptomatic patients in this study. Both asymptomatic patients and aggregative transmission are highly secretive, which make it very difficult to find the initial infected person, and easily lead to the spread of the virus in a large range. It showed that asymptomatic patients and aggregative transmission may become the main ways of rapid spread of COVID-19 outside Hubei Province (Chan et al., 2020). Therefore, reducing population aggregation and early isolation are important means to control the large-scale spread of the virus.

The time from the onset of COVID-19 to the visit was about 4.6-5.2 d (Li et al., 2020). The results of this study were basically consistent with the above-mentioned time. The shorter the visit time is, the more conducive to the control of the epidemic situation and the treatment of the disease. Fever is the main clinical manifestation of COVID-19. However, the current clinical research showed that the proportion of fever varied from $43.8 \%$ to $98.6 \%$. Fever accounted for $75 \%$ of the first symptoms in this 
study, which was between the above data. In addition, cough is the most common symptom of COVID-19, but gastrointestinal symptoms are rare, which are different from the previous characteristics of gastrointestinal symptoms after SARS-COV and MERS-COV infection (Assiri et al., 2013; Minodier et al., 2015). Although no gastrointestinal symptoms were found in this study, several clinical studies have shown that COVID-19 can cause gastrointestinal reactions such as diarrhea or vomiting (Huang et al., 2020). Therefore, in clinical work, COVID-19 cannot be simply diagnosed by fever or cough symptoms.

In patients with COVID-19 infection, the levels of leukocytes in the peripheral blood are mostly normal or decreased, and the lymphocyte count is often decreased, which can be decreased progressively with the aggravation of the disease (Huang et al., 2020; Lu et al., 2020b). In this study, the leukocyte counts of $22.5 \%$ of patients and the lymphocyte counts of $45 \%$ of patients were decreased, which may be related to the fact that most of the patients in our study are ordinary type and mild type. However, the levels of CRP and SAA were respectively increased in 55\% and $72.5 \%$ of patients with COVID-19, but PCT level was almost all normal in our study. Some studies have shown that SAA level can rise rapidly after virus infection, while CRP and PCT have no obvious response to virus infection (Chen et al., 2020). Therefore, the combined detection of SAA, PCT and CRP has a certain suggestive effect on the diagnosis of viral pneumonia (Zhu et al., 2014). The imaging manifestations of COVID-19 can be manifested as multiple patchy ground glass shadows and consolidation shadows in both lungs, mostly distributed along the bronchovascular bundle and under the pleura. There are thickening vascular shadows in lungs, which are shown as fine grid shadows with "paving stone sign", thin ground glass shadows, and local ground glass shadows around small vessels (Chinese Medical Association Radiology Branch. et al., 2020). In this study, $72.5 \%$ of patients with COVID-19 showed ground glass lesions of one or two lungs on chest CT, which was consistent with the early imaging features of COVID-19 described in previous report. At present, the gold standard for diagnosis of COVID-19 is still positive for nucleic acid test. In an analysis of 167 patients with initial diagnosis of COVID-19, the coincidence rate between positive nucleic acid test and chest CT diagnosis is $93 \% .4 \%$ of patients are positive for nucleic acid test and have clinical manifestations and exposure history, but there is no abnormality in chest CT examination. In this study, the coincidence rate of nucleic acid test positive and chest CT diagnosis is $92.5 \%$. Therefore, chest CT, as an important examination method of COVID-19, plays an important role in the detection of lesions, the judgment of the nature of lesions, the evaluation of disease conditions, clinical typing, etc., but it cannot be used as a diagnostic method of COVID-19 (Chinese Medical Association Radiology Branch. et al., 2020).

At present, there is no effective drug treatment plan for COVID-19. In previous study, treatment with antibiotics, hormones (methylprednisolone) and antiviral (oseltamivir) did not significantly improve the symptoms of patients and increase the transfer out rate of critically ill patients from ICU. All the patients in this study had stable conditions in the course of treatment, and 14 patients were cured and discharged from the hospital., suggesting that our treatment plan may have certain effect on COVID-19. Previous study has shown that prophylactic antibiotics can be used appropriately in patients with severe viral pneumonia, especially in patients who are infected after large doses of hormone (Zhong \& Zeng, 2003). Improper use of antibiotics may increase the risk of complex infections, such as fungal infections, and increase the incidence of drug resistance (Kim et al., 2011; Schauwvlieghe et al., 2018). At present, for patients with COVID-19, WHO recommends that empirical antimicrobial therapy should be based on clinical diagnosis (World Health Organization, 2020). In this study, $62.5 \%$ of the patients used antibiotics in the early stage of diagnosis and treatment, which may be related to the lack of understanding of this disease and the habit of previous empirical medication in doctors.

\section{Conclusion}

COVID-19 has the characteristics of strong infectivity, obvious epidemiological characteristics and family clustering infection. Our results suggested that asymptomatic infection may be the main way of infection for people outside Hubei Province. The most common clinical manifestation of COVID-19 is fever, but it is not the only clinical manifestation. Therefore, the diagnosis and treatment should be combined with the epidemiological history and imaging findings to avoid missed diagnosis. The detection of nucleic acid is the gold standard for the diagnosis of COVID-19, and chest CT has an effect for the diagnosis of COVID-19, but it cannot replace nucleic acid detection. At present, there is no specific drug treatment for COVID-19. It showed that the infected people outside Hubei Province may be mainly mild and ordinary types in this study, so that the application of the treatment plan in our study can effectively control the disease and improve the cure rate.

\section{Acknowledgements}

None.

\section{References}

Assiri, A., McGeer, A., Perl, T. M., Price, C. S., Al Rabeeah, A. A., Cummings, D. A., Alabdullatif, Z. N., Assad, M., Almulhim, A., Makhdoom, H., Madani, H., Alhakeem, R., Al-Tawfiq, J. A., Cotten, M., Watson, S. J., Kellam, P., Zumla, A. I., \& Memish, Z. A., and the KSA MERS-CoV Investigation Team (2013). Hospital outbreak of Middle East respiratory syndrome Coronavirus. The New England Journal of Medicine, 369(5), 407-416. http://dx.doi.org/10.1056/ NEJMoa1306742. PMid:23782161.

Bogoch II, Watts A, Thomas-Bachli A, Huber C, Kraemer MUG, Khan K. (2020). Potential for global spread of a novel coronavirus from China. Journal of Travel Medicine, 27(2):1-5.

Chan, J. F., Yuan, S., Kok, K. H., To, K. K., Chu, H., Yang, J., Xing, F., Liu, J., Yip, C. C., Poon, R. W., Tsoi, H. W., Lo, S. K., Chan, K. H., Poon, V. K., Chan, W. M., Ip, J. D., Cai, J. P., Cheng, V. C., Chen, H., Hui, C. K., \& Yuen, K. Y. (2020). A familial cluster of pneumonia associated with the 2019 novel coronavirus indicating person-to-person transmission: a study of a family cluster. Lancet, 395(10223), 514-523. http://dx.doi.org/10.1016/S0140-6736(20)30154-9. PMid:31986261.

Chen, N., Zhou, M., Dong, X., Qu, J., Gong, F., Han, Y., Qiu, Y., Wang, J., Liu, Y., Wei, Y., Xia, J., Yu, T., Zhang, X., \& Zhang, L. (2020). 
Epidemiological and clinical characteristics of 99 cases of 2019 novel coronavirus pneumonia in Wuhan, China: a descriptive study. Lancet, 395(10223), 507-513. http://dx.doi.org/10.1016/ S0140-6736(20)30211-7. PMid:32007143.

Chinese Medical Association Radiology Branch. (2020). Radiological diagnosis of new coronavirus pneumonia: Expert recommendations of chinese medical association radiology branch. Chinese Journal of Radiology, 54,E001-E001.

Drosten, C., Günther, S., Preiser, W., van der Werf, S., Brodt, H. R., Becker, S., Rabenau, H., Panning, M., Kolesnikova, L., Fouchier, R. A., Berger, A., Burguière, A. M., Cinatl, J., Eickmann, M., Escriou, N., Grywna, K., Kramme, S., Manuguerra, J. C., Müller, S., Rickerts, V., Stürmer, M., Vieth, S., Klenk, H. D., Osterhaus, A. D., Schmitz, H., \& Doerr, H. W. (2003). Identification of a novel coronavirus in patients with severe acute respiratory syndrome. The New England Journal of Medicine, 348(20), 1967-1976. http://dx.doi.org/10.1056/ NEJMoa030747. PMid:12690091.

Huang, C., Wang, Y., Li, X., Ren, L., Zhao, J., Hu, Y., Zhang, L., Fan, G., Xu, J., Gu, X., Cheng, Z., Yu, T., Xia, J., Wei, Y., Wu, W., Xie, X., Yin, W., Li, H., Liu, M., Xiao, Y., Gao, H., Guo, L., Xie, J., Wang, G., Jiang, R., Gao, Z., Jin, Q., Wang, J., \& Cao, B. (2020). Clinical features of patients infected with 2019 novel coronavirus in Wuhan, China. Lancet, 395(10223), 497-506. http://dx.doi.org/10.1016/ S0140-6736(20)30183-5. PMid:31986264.

Kim, S. H., Hong, S. B., Yun, S. C., Choi, W. I., Ahn, J. J., Lee, Y. J., Lee, H. B., Lim, C. M., \& Koh, Y. (2011). Corticosteroid treatment in critically ill patients with pandemic influenza A/H1N1 2009 infection: analytic strategy using propensity scores. American Journal of Respiratory and Critical Care Medicine, 183(9), 1207-1214. http:// dx.doi.org/10.1164/rccm.201101-0110OC. PMid:21471084.

Kuiken, T., Fouchier, R. A. M., Schutten, M., Rimmelzwaan, G. F., van Amerongen, G., van Riel, D., Laman, J. D., de Jong, T., van Doornum, G., Lim, W., Ling, A. E., Chan, P. K., Tam, J. S., Zambon, M. C., Gopal., R., Drosten, C., van der Werf, S., Escriou, N., Manuguerra, J. C., Stöhr, K., Peiris, J. S., \& Osterhaus, A. D. (2003). Newly discovered coronavirus as the primary cause of severe acute respiratory syndrome. Lancet, 362(9380), 263-270. http://dx.doi.org/10.1016/ S0140-6736(03)13967-0. PMid:12892955.

Li, Q., Guan, X., Wu, P., Wang, X., Zhou, L., Tong, Y., Ren, R., Leung, K. S. M., Lau, E. H. Y., Wong, J. Y., Xing, X., Xiang, N., Wu, Y., Li, C., Chen, Q., Li, D., Liu, T., Zhao, J., Liu, M., Tu, W., Chen, C., Jin, L., Yang, R., Wang, Q., Zhou, S., Wang, R., Liu, H., Luo, Y., Liu, Y., Shao, G., Li, H., Tao, Z., Yang, Y., Deng, Z., Liu, B., Ma, Z., Zhang, Y., Shi, G., Lam, T. T. Y., Wu, J. T., Gao, G. F., Cowling, B. J., Yang, B., Leung, G. M., \& Feng, Z. (2020). Early transmission dynamics in Wuhan, China, of novel coronavirus-infected pneumonia. The New England Journal of Medicine, 382(13), 1199-1207. http://dx.doi. org/10.1056/NEJMoa2001316. PMid:31995857.
Lu, H., Stratton, C. W., \& Tang, Y. W. (2020a). Outbreak of pneumonia of unknown etiology in Wuhan China: the mystery and the miracle. Journal of Medical Virology, 92(4), 401-402. http://dx.doi.org/10.1002/ jmv.25678. PMid:31950516.

Lu, R., Zhao, X., Li, J., Niu, P., Yang, B., Wu, H., Wang, W., Song, H., Huang, B., Zhu, N., Bi, Y., Ma, X., Zhan, F., Wang, L., Hu, T., Zhou, H., Hu, Z., Zhou, W., Zhao, L., Chen, J., Meng, Y., Wang, J., Lin, Y., Yuan, J., Xie, Z., Ma, J., Liu, W. J., Wang, D., Xu, W., Holmes, E. C., Gao, G. F., Wu, G., Chen, W., Shi, W., \& Tan, W. (2020b). Genomic characterization and epidemiology of 2019 novel coronavirus: implications of virus origins and receptor binding. Lancet, 395(10224), 565-574. http://dx.doi.org/10.1016/S0140-6736(20)30251-8.

Minodier, L., Charrel, R. N., Ceccaldi, P. E., van der Werf, S., Blanchon, T., Hanslik, T., \& Falchi, A. (2015). Prevalence of gastrointestinal symptoms in patients with influenza, clinical significance, and pathophysiology of human influenza viruses in faecal samples: what do we know? Virology Journal., 12(1), 215. http://dx.doi.org/10.1186/ s12985-015-0448-4. PMid:26651485.

Schauwvlieghe, A., Rijnders, B., Philips, N., Verwijs, R., Vanderbeke, L., Van Tienen, C., Lagrou, K., Verweij, P. E., Van de Veerdonk, F. L., Gommers, D., Spronk, P., Bergmans, D. C. J. J., Hoedemaekers, A., Andrinopoulou, E. R., van den Berg, C. H. S. B., Juffermans, N. P., Hodiamont, C. J., Vonk, A. G., Depuydt, P., Boelens, J., \& Wauters, J. (2018). Invasive aspergillosis in patients admitted to the intensive care unit with severe influenza: a retrospective cohort study. The Lancet. Respiratory Medicine, 6(10), 782-792. http://dx.doi.org/10.1016/ S2213-2600(18)30274-1. PMid:30076119.

World Health Organization - WHO (2020). Clinical management of severe acute respiratory infection when novel coronavirus ( $\mathrm{CoV}$ ) infection is suspected. Geneva: World Health Organization. Retrieved from https://www.who.int/publications-detail/clinical-management-ofsevere-acute-respiratory-infection-when-novelcoronavirus-(ncov)infection-is-suspected

Zhong, N., \& Zeng, G. (2003). Our strategies forfighting Severe Acute Respiratory Syndrome (SARS). American Journal of Respiratory and Critical Care Medicine, 168(1), 7-9. http://dx.doi.org/10.1164/ rccm.200305-707OE. PMid:12773318.

Zhu, N., Zhang, D., Wang, W., Li, X., Yang, B., Song, J., Zhao, X., Huang, B., Shi, W., Lu, R., Niu, P., Zhan, F., Ma, X., Wang, D., Xu, W., Wu, G., Gao, G. F., \& Tan, W. (2020). A Novel Coronavirus from Patients with Pneumonia in China. The New England Journal of Medicine, 382(8), 727-733. http://dx.doi.org/10.1056/NEJMoa2001017. PMid:31978945.

Zhu, X., Yong, D., \& Huang, G. (2014). Differential effect of PCT, hsCRP, SAA on bacterial and viral infection. International Journal of Laboratory Medicine, 35,3048-3050. 